\title{
ARISTOLOCHIA EMILIAE (ARISTOLOCHIACEAE: SUBSECCIÓN PENTANDRAE), UNA ESPECIE NUEVA DE LA COSTA DE JALISCO, MÉXICO
}

\author{
Francisco J. Santana-Michel y José Arturo Solís-Magallanes \\ Universidad de Guadalajara, Centro Universitario de la Costa Sur \\ Departamento de Ecología y Recursos Naturales \\ Independencia Nacional 151, Apdo. postal 64 \\ 48900 Autlán, Jalisco, México. fsantanam@cucsur.udg.mx
}

\section{RESUMEN}

Se describe e ilustra a Aristolochia emiliae, procedente de la Estación de Investigación, Experimentación y Difusión "Chamela" en el municipio de La Huerta, Jalisco. La planta crece en el bosque tropical caducifolio. Se caracteriza por presentar flores geniculadas menores de $3 \mathrm{~cm}$ de largo, limbo del cáliz orbicular con pelos uncinados, hojas trilobadas con los lóbulos basales ancoriformes; esta última característica es única en las especies mexicanas de la subsección Pentandrae.

Palabras clave: Aristolochia, Aristolochiaceae, Chamela, Jalisco, Pentandrae, taxonomía.

\begin{abstract}
Aristolochia emiliae sp. nov., found in tropical deciduous forest in the Estación de Investigación, Experimentación y Difusión "Chamela", municipality of La Huerta, Jalisco, Mexico, is described and illustrated. The geniculate flowers are less than $3 \mathrm{~cm}$ long with uncinate hairs on the orbicular calyx limb. The basal lobes of the trilobate leaves are anchorshaped, a unique characteristic among the Mexican species in the Pentandrae subsection of Aristolochia.
\end{abstract}

Key words: Aristolochia, Aristolochiaceae, Chamela, Jalisco, Pentandrae, taxonomy. 
El género Aristolochia L. comprende la mayor diversidad de especies de la familia Aristolochiaceae (González, 1997) y de su sección Gymnolobus Duchartre, la subsección Pentandrae Duchartre, está representada en su mayoría por especies de México (Duchartre, 1854, 1864; Pfeifer, 1970).

Los inventarios regionales de plantas vasculares en los estados de Colima y Jalisco han aportado para el caso de la familia Aristolochiaceae, una considerable cantidad de registros nuevos y por lo menos cuatro especies nuevas para la ciencia. Para el inventario de la región de Chamela (Lott, 1993 ), Emily Lott y Arturo Solís Magallanes colectaron una especie de Aristolochia que fue identificada como $A$. aff. variifolia Duchr. Al revisar los ejemplares nos dimos cuenta que se trata de una especie nueva para la ciencia y su descripción es la siguiente:

Aristolochia emiliae Santana-Michel et Solís-Magallanes sp. nov., Figs. 1 y 2.

Plantae perennes erectae vel in maturitate prostratae $25-70 \mathrm{~cm}$ longae: folia apice acuminata, lamina trilobata, $(2.5-) 5-8.5(-10) \mathrm{cm}$ longa, $(1-) 1.5-3.5(-4.5) \mathrm{cm}$ lata, lobis basalibus quam centrali brevioribus, ancoriformibus; calyx geniculatus ( $180^{\circ}$ flexus), 2-2.8 cm longus, 2-2.5 cm latus, limbo 1-lobato, 2-3.5 cm longo, 2-2.2 cm lato, orbiculari, purpureo, piloso pilis uncinatis $0.3-0.4 \mathrm{~mm}$ longis, tubo tubuloso flexo 1.3-2.1 cm longo, 4-5 mm diametro.

Plantas perennes, erectas a postradas, en la madurez de 25-70 cm de largo; hojas pecioladas, ápice acuminado, haz esparcidamente piloso a glabrescente, envés glabrescente, piloso entre las nervaduras y los bordes, peciolo corto, de (0.3-)0.4$0.9(-1) \mathrm{cm}$ de largo, 0.4-0.5 mm de diámetro, lámina trilobada, de (2.5-)5-8.5(-10) cm de largo, (1-)1.5-3.5(-4.5) cm de ancho, los lóbulos basales en forma de ancla, más cortos que el lóbulo central; ramas floríferas postradas con hojas reducidas, saliendo de la base de los tallos; flores solitarias, axilares, el pedúnculo bracteolado, piloso, de 5-8 $\mathrm{mm}$ de largo, $1 \mathrm{~mm}$ de diámetro, bracteolas lanceoladas, pilosas en ambas superficies, de 5-8 $\mathrm{mm}$ de largo, 3-5 $\mathrm{mm}$ de ancho, cáliz geniculado (doblado $180^{\circ}$ ), de 2-2.8 cm de largo, 2-2.5 cm de ancho, limbo 1-lobado, de 2-3.5 cm de largo, 2-2.2 $\mathrm{cm}$ de ancho, orbicular, de color púrpura, piloso, los pelos uncinados, de 0.3-0.4 mm de largo, garganta oblata, glabra, del mismo color que el limbo, hipantio no evidente, utrículo elipsoide, 8-9-nervado, piloso sobre las nervaduras, de 9-11 mm de largo, 8-9 mm de diámetro, siringe excéntrica, tubular, de 3-3.5 $\mathrm{mm}$ de largo, 2-2.5 $\mathrm{mm}$ de diámetro, tubo tubular, doblado, esparcidamente piloso, de 1.3-2.1 cm de largo, 4-5 $\mathrm{mm}$ de diámetro, ginostemo 5-lobado, de 3-3.5 mm de largo, 2.8-3 mm de diámetro, 
Santana-Michel y Solís-Magallanes: Aristolochia emiliae, especie nueva de Jalisco

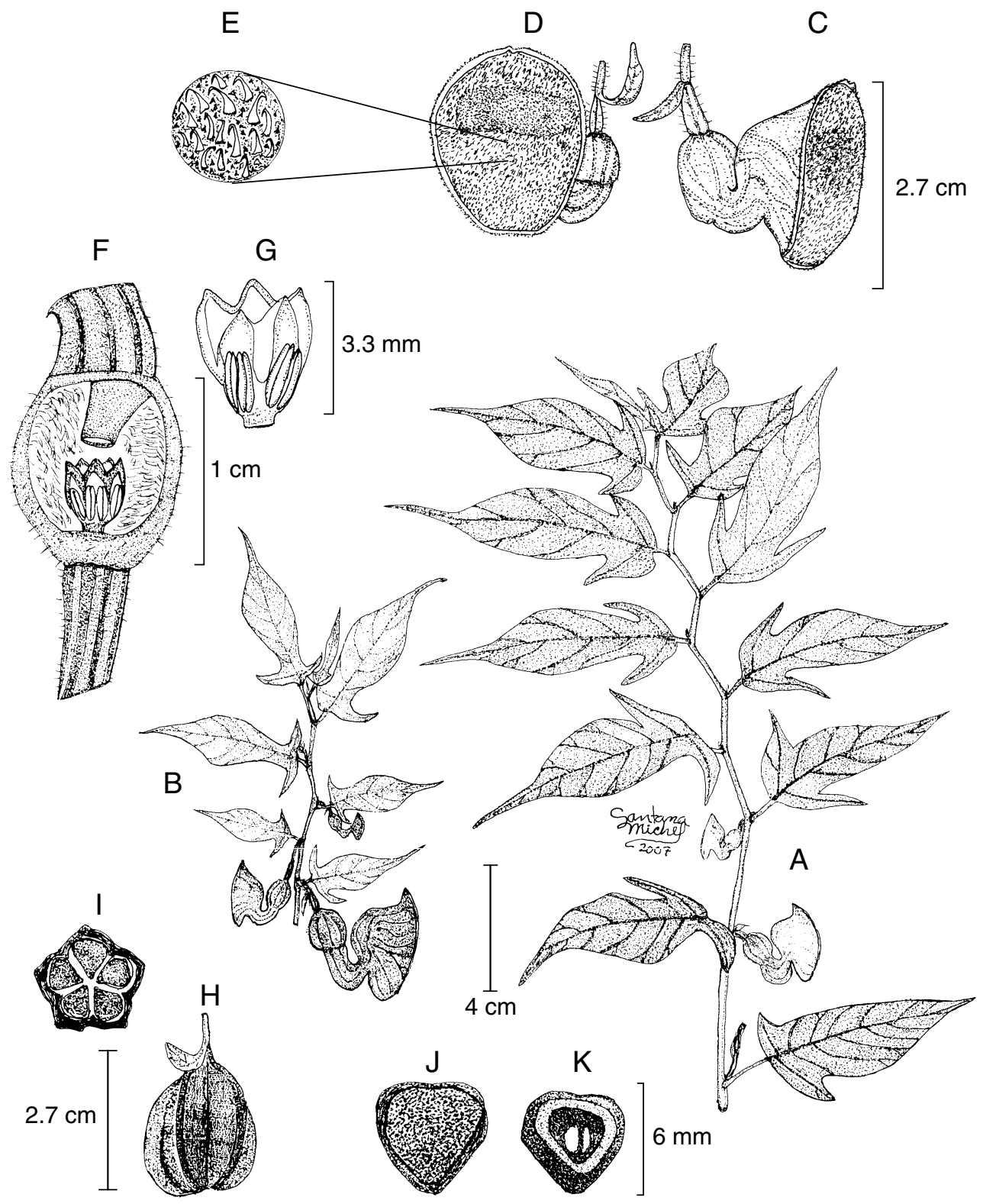

Fig. 1. Aristolochia emiliae. A. rama erecta de la planta; B. rama florífera postrada; C. flor vista de costado; D. flor vista de frente; E. detalle de la superficie del limbo; F. utrículo mostrando la siringe y el ginostemo; G. ginostemo; H. fruto; I. corte trasversal del fruto; J y K. semilla. Ilustración basada en la colección tipo (F. J. Santana-Michel 9238). 

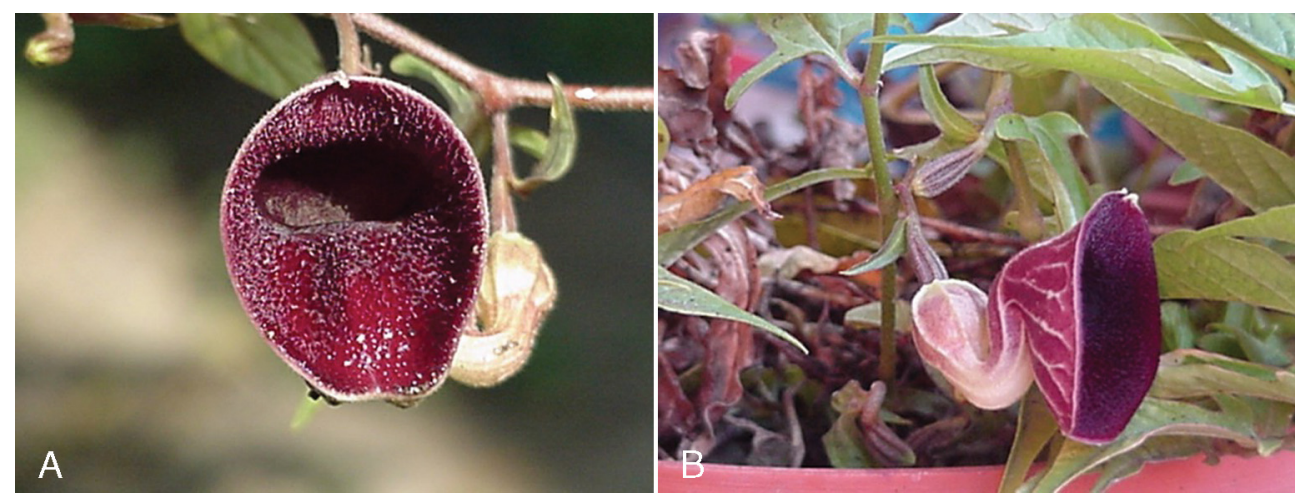

Fig. 2. Flor de Aristolochia emiliae vista de frente (A) y de costado (B).

lóbulos de 1-1.5 mm de largo, estípite de 0.5-1 mm de largo, estambres 5, anteras de 1.5-2 $\mathrm{mm}$ de largo, $1 \mathrm{~mm}$ de ancho; fruto una cápsula ovoide 5-carpelar, piloso, de 2-3 cm de largo, 1.7-2.5 cm de diámetro, dehiscencia basicida, semillas triangulares, numerosas, negras, de 5-6 $\mathrm{mm}$ de largo, 4.5-5.5 $\mathrm{mm}$ de ancho, $1 \mathrm{~mm}$ de grueso, superficie finamente tuberculada.

Tipo: México, Jalisco, municipio de La Huerta, Estación de Investigación, Experimentación y Difusión Chamela, UNAM, en la Vereda El Búho antes del Arroyo El Zarco, altitud 180 m s.n.m., bosque tropical caducifolio: Heliocarpus, Cordia, Thouinia, Crescentia, Ficus, Spondias, Croton, Lysiloma, Jatropha, Bernardia, Randia, Lonchocarpus, Trichilia, 11 de noviembre de 1998, F. J. Santana-Michel 9238 (holótipo depositado en el herbario ZEA; isótipos por distribuirse a los herbarios ENCB, IBUG, IEB, MEXU).

Material adicional examinado: México, Jalisco, municipio La Huerta, Estación de Investigación, Experimentación y Difusión Chamela, UNAM, 150 m s.n.m., bosque tropical caducifolio, 29 de septiembre de 1982, J. A. Solís-Magallanes 3849 (MEXU, UCR); ibid., Emily J. Lott 1532; 1699 (MEXU).

Etimología: el nombre de esta especie se dedica a Emily J. Lott por sus importantes contribuciones a la flora de la región costera de Jalisco y Colima.

Distribución conocida: Sólo se ha colectado en la localidad tipo. 
Santana-Michel y Solís-Magallanes: Aristolochia emiliae, especie nueva de Jalisco

\begin{tabular}{|c|c|c|c|c|c|c|c|c|c|c|}
\hline 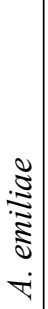 & 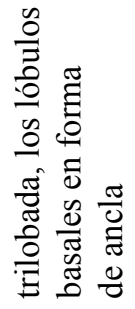 & 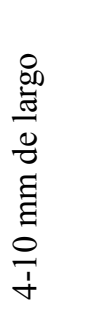 & 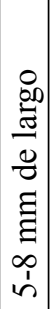 & 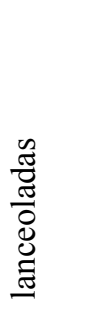 & 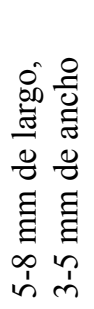 & 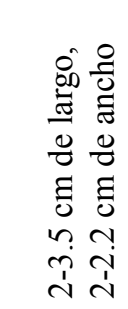 & $\begin{array}{l}\frac{\bar{\Xi}}{3} \\
\frac{0}{0} \\
\dot{0}\end{array}$ & 苛 & 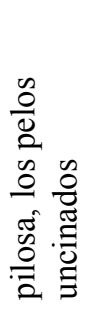 & $\frac{\frac{\pi}{\frac{\pi}{0}}}{\frac{0}{0}}$ \\
\hline 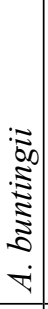 & 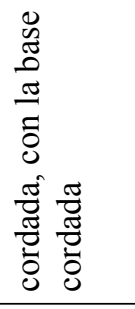 & 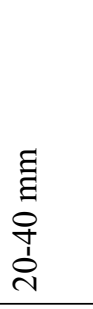 & \begin{tabular}{|c|}
$\vdots$ \\
$\vdots$ \\
$n$ \\
$n$ \\
\\
0 \\
\end{tabular} & $\begin{array}{l}\frac{\pi}{0} \\
\frac{\pi}{0} \\
0 \\
0\end{array}$ & 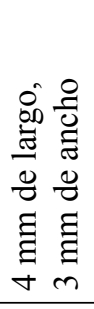 & 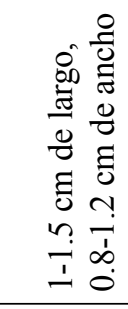 & 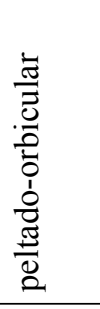 & .0: & 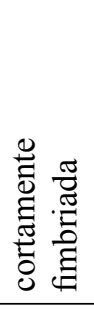 & $\begin{array}{l}\frac{\pi}{\sigma} \\
\frac{\pi}{2} \\
0\end{array}$ \\
\hline 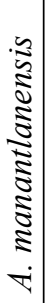 & 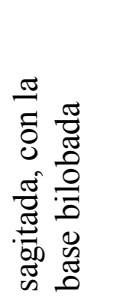 & 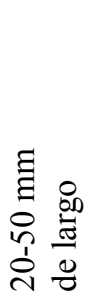 & 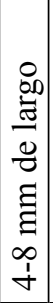 & 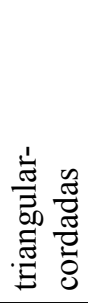 & 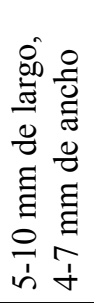 & 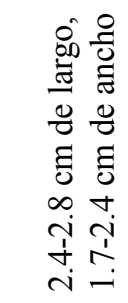 & 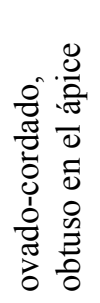 & 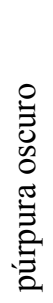 & 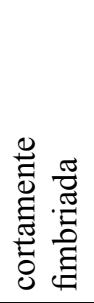 & 离 \\
\hline 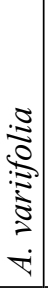 & 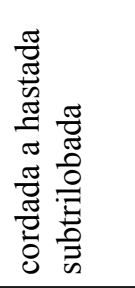 & 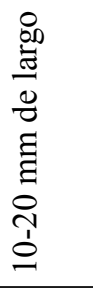 & 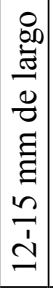 & 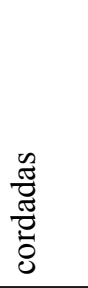 & 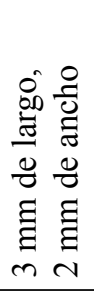 & 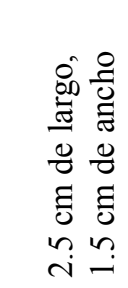 & 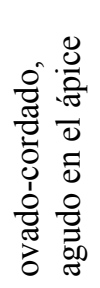 & 苂 & 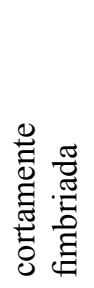 & $\frac{\bar{\Xi}}{\bar{J}}$ \\
\hline 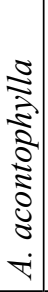 & 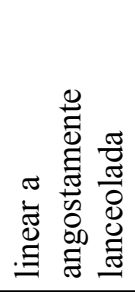 & $\begin{array}{l}8 \\
00 \\
\frac{\Xi}{0} \\
0 \\
\Xi \\
\Xi \\
0 \\
0 \\
\dot{y}\end{array}$ & $\begin{array}{c}0 \\
00 \\
\vdots \\
0 \\
0 \\
\vdots \\
\vdots \\
\vdots \\
n \\
\\
\\
\end{array}$ & 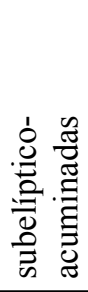 & 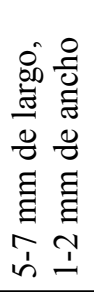 & 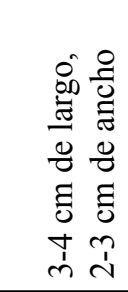 & 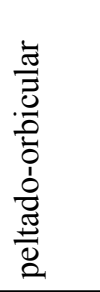 & 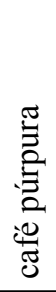 & 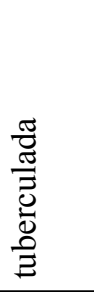 & 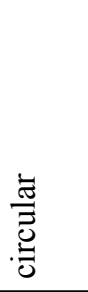 \\
\hline & 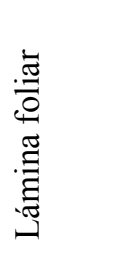 & $\begin{array}{l}\frac{0}{0} \\
\frac{0}{0} \\
0 \\
0\end{array}$ & 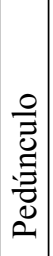 & 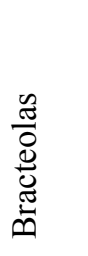 & 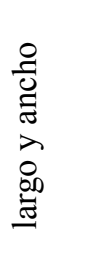 & 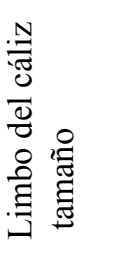 & 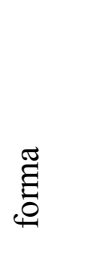 & $\frac{\dot{0}}{8}$ & 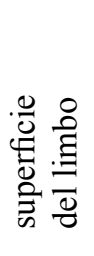 & 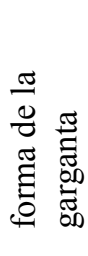 \\
\hline
\end{tabular}


Época de floración: de septiembre a noviembre.

Hábitat: Bosque tropical caducifolio con las siguientes especies arbóreas: $L y$ siloma microphyllum, Bursera instabilis, B. heteresthes, Jatropha malacophylla, J. standleyi, Croton pseudoniveus, Cordia alliodora, Heliocarpus pallidus, Trichilia trifolia ssp. palmeri, Lonchocarpus eriocarinalis, Guettarda elliptica, Spondias purpurea, Caesalpinia coriaria, Ceiba aesculifolia, Thouinia paucidentata, Ficus cotinifolia, Bernardia spongiosa, Crescentia alata.

Aristolochia emiliae, de acuerdo con el trabajo monográfico de Pfeifer (1970), pertenece a la subsección Pentandrae, en el grupo de las especies con flores geniculadas con el tubo doblado $180^{\circ}$ y cálices de menos de $3 \mathrm{~cm}$ de largo. Descuella por su limbo calicino orbicular con pelos uncinados, así como por su lámina foliar trilobada, con los lóbulos basales en forma de ancla, rasgo único entre las especies mexicanas de la mencionada subsección.

Por las características de sus flores Aristolochia emiliae se encuentra relacionada con A. acontophylla Pfeifer, A. variifolia Duch., A. manantlanensis SantanaMichel (Santana-Michel, 2007) y A. buntingii Pfeifer. En el Cuadro 1 se muestran las diferencias entre estas especies.

\section{AGRADECIMIENTOS}

Agradecemos al Dr. Jerzy Rzedowski Rotter, quien amablemente colaboró en la diagnosis en latín y atinados comentarios; al Dr. Felipe A. Noguera Martínez por su apoyo durante nuestra visita a la Estación de Investigación, Experimentación y Difusión Chamela de la Universidad Nacional Autónoma de México. Asimismo, damos las gracias a las autoridades del Centro Universitario de la Costa Sur de la Universidad de Guadalajara y compañeros del Laboratorio de Botánica por sus diversas formas de apoyo.

\section{LITERATURA CITADA}

Duchartre, P. 1854. Tentamen methodicae divisionis generis Aristolochia. Ann. Sci. Nat., Bot. Ser. 4(2): 29-76.

Duchartre, P. 1864. Aristolochiaceae. In: A. De Candolle. Prodromus 15(1): 421-498. 
González, F. 1997. Hacia una filogenia de Aristolochia y sus congéneres neotropicales. Caldasia 19(1-2): 115-130.

Lott, E. J. 1993. Annotated checklist of the vascular flora of the Chamela bay region, Jalisco, Mexico. Occas. Pap. Calif. Acad. Sci. 148: 60.

Pfeifer, H. W. 1970. A revision of the pentandrous species of Aristolochia. The University of Connecticut Publication Series. Storrs, Connecticut. $134 \mathrm{pp}$.

Santana-Michel, F. J. 2007. Una especie nueva de Aristolochia L., subsección Pentandrae (Aristolochiaceae) de la reserva de la biosfera Sierra de Manantlán, Jalisco, México. Acta Bot. Mex. 79: 81-87.

Recibido en febrero de 2007.

Aceptado en agosto de 2007. 
